\title{
Treatment of Facial Neuralgia Developed after Inferior Meatal Antrostomy by Narrowing of the Inlet with Endoscopic Cartilage Graft
}

\author{
Jong Sook Yi, MD and Hong Ryul Jin, MD \\ Doctor Jin's Premium Nose Clinic, Seoul, Korea
}

Inferior meatal antrostomy (IMA) is a widely performed surgical technique to treat postoperative maxillary mucocele. The method is safe and easy to perform, without major complications compared with other approaches. Facial pain after IMA is a rare clinical entity that can be challenging to diagnose and treat. The authors present an unusual case of acute facial neuralgia triggered by cold air that developed after IMA. The antrostomy was located at the anterior-most part of the inferior meatus, and the inlet size was relatively large compared with the size of the remaining sinus. Surgical narrowing of the antrostomy inlet using endoscopy dramatically reduced the symptoms, and symptom relief was maintained for up to one year after surgery.

KEY WORDS: Facial pain · Caldwel-Luc operation · Inferior meatal antrostomy · Inferior orbital nerve dehiscence.

\section{INTRODUCTION}

Transnasal endoscopic marsupialization via inferior meatal antrostomy (IMA) and middle meatal antrostomy (MMA) is gaining popularity as a standard surgical method to treat postoperative maxillary mucocele. Although MMA is more physiologic, IMA is preferred because the mucocele is mostly located near the lower part of the lateral nasal wall, with bulging into the nasal cavity. Even with contracted and fibrotic sinus, which is common after the Caldwell-Luc (C-L) operation, IMA can be performed easily and effectively without major complications, such as bleeding, facial numbness, and nasolacrimal duct injury. ${ }^{1-5)}$

The authors experienced a rare case of facial neuralgia that developed after IMA to treat postoperative maxillary mucocele. The patient was successfully treated by surgically narrowing the IMA ostium. The diagnosis, surgical method, results, and possible pathophysiologic mechanism are discussed.

\section{CASE REPORT}

A 52-year-old woman reported a 6-year history of leftsided facial and ocular pain that developed after left-sided IMA to treat postoperative mucocele. She had undergone a C-L operation 20 years earlier. The pain was sharp and electric aggravated in cold air. When there's acute pain attack, visual analogue score (VAS) was 9 out of 10. Previously she had tried conservative treatment for years including pain reliever, intranasal ointment, and saline irrigation of nasal cavity which was unsuccessful. Anticonvulsant drug responded a little but couldn't keep taking it because of side effect. Instead, her nasal symptom was much relieved when the nostril was partially blocked with cotton pledget. She did not report other rhinologic symptoms, such as nasal obstruction, rhinorrhea, or hyposmia. Endoscopic examination showed a patent antrostomy inlet, about $1.0 \mathrm{~cm}$ in diameter, at the anterior-most part of the left inferior meatus. The shape and mucosa of inferior turbinate were intact, and the nasal cavity was normal. OMU computed tomography (CT) scan

Received: January 15, 2019 / Revised: February 27, 2019 / Accepted: April 29, 2019

Address for correspondence: Hong Ryul Jin, MD, PhD, Doctor Jin's Premium Nose Clinic, 621 Gangnam-daero, Seocho-gu, Seoul 06524, Korea Tel: +82-2-2088-8585, Fax:+82-2-2088-6543, E-mail: doctorjin@daum.net 
showed a contracted, small maxillary sinus due to new bone formation and fibrosis and a relatively large antrostomy (Fig. 1). The infraorbital nerve (ION) canal was sagging downward in the contracted sinus. Because she demonstrated pain relief with nasal blockage, we planned to narrow the IMA inlet to prevent cold air from directly entering and stimulating the sinus wall.

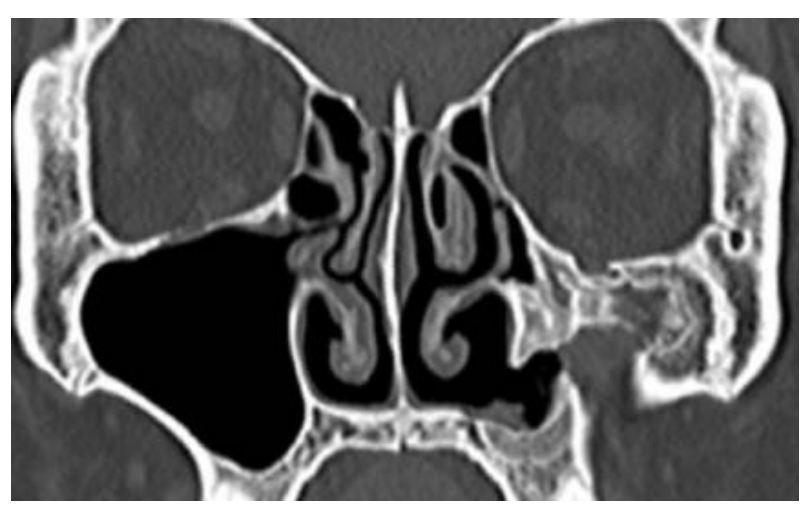

Fig. 1. OMU CT scan showed small, contracted left maxillary sinus with fibrosis and new bone formation. The antrostomy inlet is relatively large compared to the small sinus.
Operation (Fig. 2). An inferior vestibular incision was made in the left nostril from floor to lateral wall. The submucosal flap was elevated to the antrostomy inlet while preserving mucosa and skin integrity. Meticulous dissection was done around the antrostomy anteriorly, inferiorly, and superiorly. Harvested septal cartilage and conchal cartilage pieces were inserted into the submucosal pocket to obstruct the antrostomy inlet, with approximately $80 \%$ obstruction achieved.

The patient immediately noticed improvement of symptoms after the operation (VAS score from 9 to 2) and this improvement persisted thereafter. She could breathe painlessly even in cold weather without nostril blockage with much decreased pain. On endoscopic examination, wellmaintained IMA inlet narrowing was observed at 6 months after operation (Fig. 3). We believe this is the first case report of successful treatment of facial neuralgia that developed after IMA.
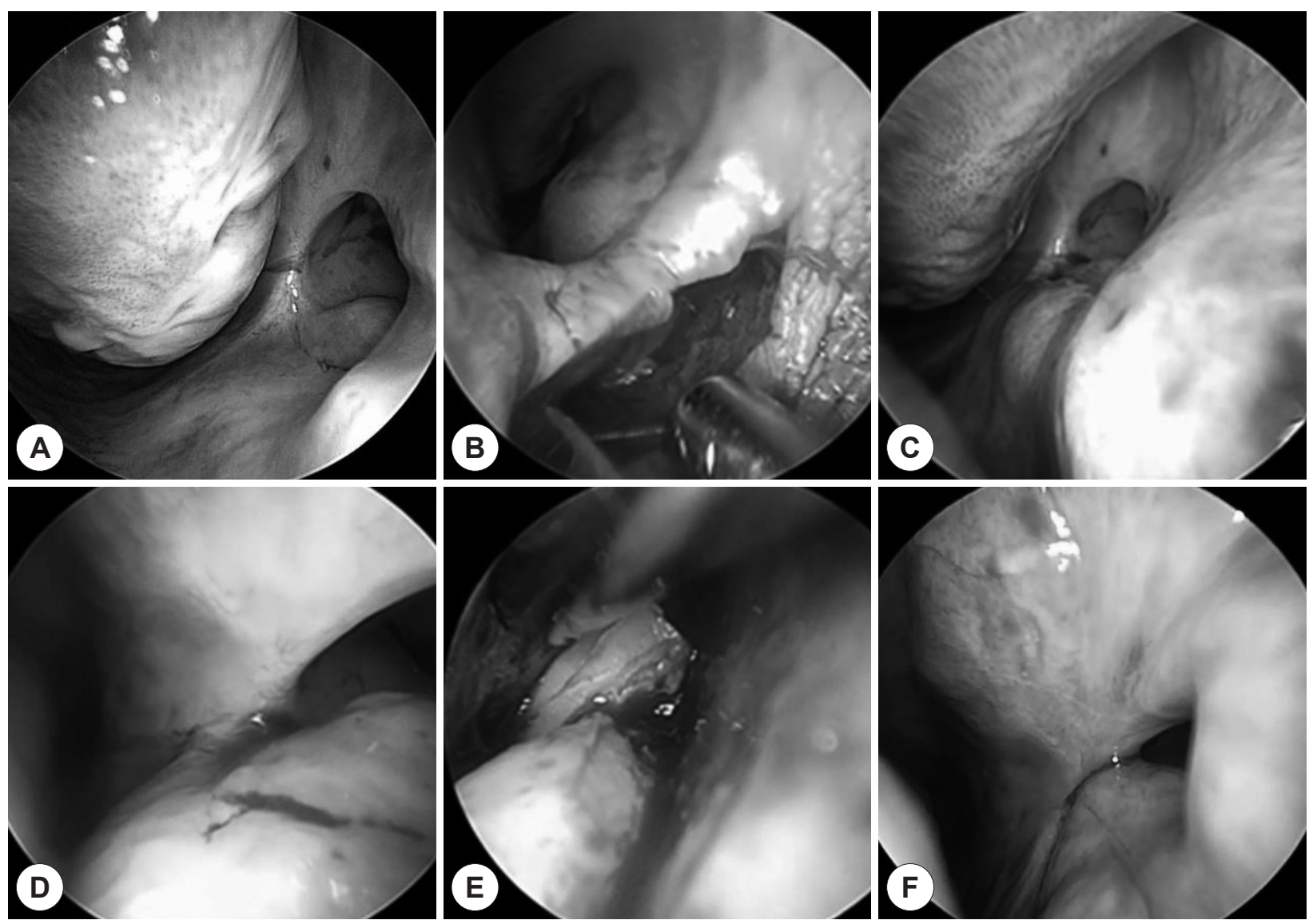

Fig. 2. IMA site reconstruction. A: About $1.0 \mathrm{~cm}$ sized, patent antrostomy at the anterior part of the left inferior meatus, B: endoscopic vestibular incision, C, D: dissection of pocket, E: insertion of cartilage chips, F: ostium narrowing after surgery. 

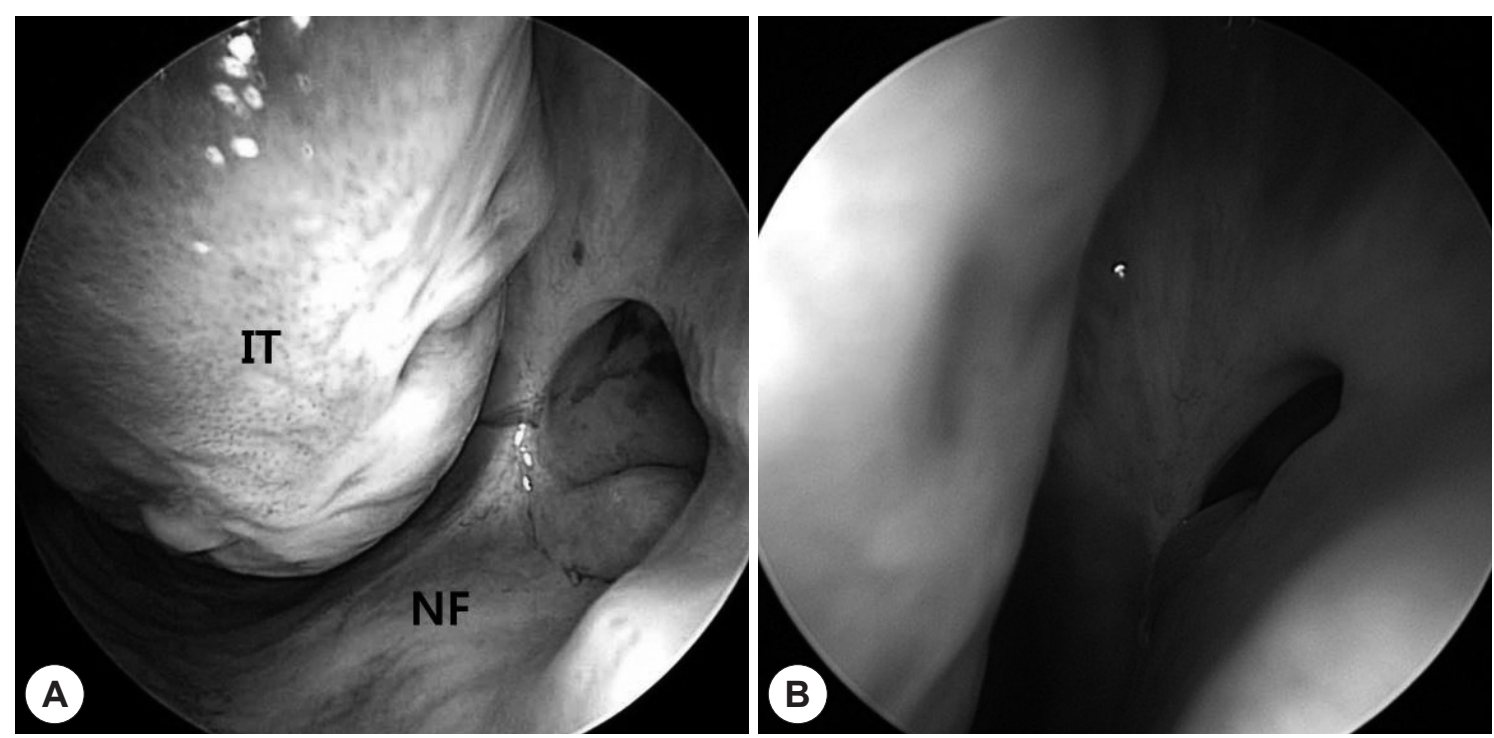

Fig. 3. Pre (A) and postoperative 6 months (B) endoscopic view of the IMA opening. Narrowed antrostomy inlet was well maintained. IT: inferior turbinate, NF: nasal floor

\section{DISCUSSION}

Considering the character of the patient's pain — sharp, shock like, and sudden - we could assume that the pain is neuropathic in origin. Trigeminal neuralgia is uncommon disorder characterized by recurrent attacks of lancinating pain in the nerve distribution, and brief attacks are triggered by talking, chewing, teeth brushing, or cold breeze typically. ${ }^{6}$ The pain is nearly always unilateral and occur repeatedly throughout the day. We supposed that cold air directly entering the sinus of the patient may have triggered neuralgic pain in the left side of the face. Although cold air is known as a common triggering factor for trigeminal neuralgia, the exact pathophysiologic mechanism of how the cold ambient air caused neuralgic pain in the left side of the patient's face is not clear.

The diagnosis is typically determined clinically, so the patient's history is the most critical in the evaluation although imaging studies may be necessary to rule out other disease. ${ }^{\text {) }}$ CT scan of this patient showed the ION sagging into the sinus during its course on the orbital floor, suggesting a possible clue to the neuralgic origin of the patient's pain. The ION travels anteriorly on the orbit floor through the thin infraorbital canal and exits from the infraorbital foramen into the face, reaching the skin. Not all sinus mucosa exposed to cold air would induce facial pain, but nerve irritation may happen, especially when the nerve is exposed from the canal. According to anatomic study, ION canal thickness is only $0.2 \mathrm{~mm}$, and the incidence of partial or complete dehiscence of the infraorbital canal is between $12 \%$ and $20 \%$ in dry skull specimens. ${ }^{7-9)}$ Moreover, unexpected variations of the nerve course in the maxillary sinus or canal dehiscence can increase the possibility of iatrogenic injury during C-L surgery. The mechanism of pain development by the dehiscent infraorbital canal is still unclear. But Facial pain in the distribution of the ION is often labelled "vacuum maxillay sinusitis" and treated empirically by intranasal antrostomy to enlarge the inlet. ${ }^{10)}$ Some authors have suggested alterations in atmospheric pressure as a barotrauma, and others have suggested a vasodilation effect of the antral mucosal blood vessel. ${ }^{1112)}$ However, these assumptions are mostly related to small antral ostium size and do not correlate to our patient, who has a large IMA inlet. Sessle et al. ${ }^{13)}$ suggested that the trigeminal primary afferent fibers terminated in the nasal mucosa as a free nerve ending were activated by noxious stimuli, including mechanical and chemical irritants.

Another possible factor contributing to neuralgic pain development in our case is the location and size of the IMA opening. IMA is usually performed at the inferior portion of the inferior meatus where the maxillary cyst that developed after C-L operation bulges most. Thus, the neo-ostium location depends on the location of the cyst in general. The IMA opening tends to become smaller with fibrotic changes of the opening, although the opening is large as created initially. Lund et al. ${ }^{5)}$ reported that $53 \%$ of the IMA opening 
had closed with a mean follow-up time of 26 months, and adequate initial hole size should be as large as $1 \times 2 \mathrm{~cm}$ to prevent its closure. In this case, the neo-ostium had remained mostly open at 6 years after surgery. In addition, it was located too anteriorly which inferior turbinate anterior head was not able to block it even after outfracture. This situation allowed non-humidified, cold air to easily enter the sinus and irritate exposed nerve. Our treatment of blocking the IMA inlet was based on these theories and was successful.

Blocking the IMA inlet with conchal cartilage graft via an endonasal approach is not technically challenging. However, the mucosa needs to be protected, and the ostium should not be destroyed to prevent recurrence of mucocele. An endoscope helped us to dissect precisely around the ostium and to place grafts exactly to surround the ostium. The initial narrowing of up to $80 \%$ of the ostium size was maintained at 6 months after surgery. Slight resorption of the cartilage with widening of the narrowed ostium is expected with time, and long-term follow-up is warranted.

\section{CONCLUSION}

We report a case of successful treatment of facial neuralgia that developed after IMA by endoscopically narrowing the ostium with cartilage graft. The neuralgia was triggered by cold air directly entering the maxillary sinus, and we believe that the exposed ION was a contributing factor in the neuralgia.

\section{REFERENCES}

1) Basu MK, Rout PG, Rippin JW, Smith AJ. The post-operative maxillary cyst. Experience with 23 cases. Int J Oral Maxillofac Surg 1988; 17: 282-4.

2) Saito T, Ikeda T, Kono Y, Ohtsubo T, Noda I, Saito H. Implications of endoscopic endonasal surgery for the treatment of postoperative maxillary mucoceles. ORL J Otorhinolaryngol Relat Spec 2000;62:43-8.

3) Huang CC, Chen CW, Lee TJ, Chang PH, Chen YW, Chen YL, et al. Transnasal endoscopic marsupialization of postoperative maxillary mucoceles: middle meatal antrostomy versus inferior meatal antrostomy. Eur Arch Otorhinolaryngol 2011;268:1583-7.

4) Lee JY, Baek BJ, Byun JY, Shin JM. Long-term efficacy of inferior meatal antrostomy for treatment of postoperative maxillary mucoceles. Am J Oto Surg 2014;727-30.

5) Lund VJ. Fundamental considerations of the design and function of intranasal antrostomies. Rhinology 1985;23:231-6.

6) Rudolph M. Trigeminal neuralgia. Am Fam Physician 2008;77:1291-6.

7) Sharma N, De M, Pracy P. Recurrent facial paresthesia secondary to maxillary antral cyst and dehiscent infraorbital canal: case report. J Laryngol Otol 2007;121:e6.

8) Hollinshead WH. Anatomy for regeon v.1: the head and neck. New York: Harper \& Row;1968.

9) Gray HGCM. Anatomy of the human body. Philadelphia, PA: Lea \& Febiger;1966.

10) Whittet HB. Infraorbital nerve dehiscence: the anatomic cause of maxillary sinus "vacuum headache"? Otolaryngol Head Neck Surg 1992;107(1):21-8

11) Mirza S, Richardson H. Otic barotrauma from air travel. J Laryngol Otol 2005;119:366-370.

12) Falck B, Svanholm H, Aust R, Bäcklund L. The relationship between body posture and pressure in occluded maxillary sinus of man. Rhinology 1989;27:161-7.

13) Sessle BJ. Peripheral and central mechanisms of orofacial inflammatory pain. Int Rev Neurobiol 2011;97:179-206. 